\title{
A New Bi-Frequency Soil Smart Sensing Moisture and Salinity for Connected Sustainable Agriculture
}

\author{
Julien Roux, Christophe Escriba, Jean-Yves Fourniols, George Soto-Romero \\ LAAS-CNRS, Université de Toulouse, CNRS, INSA, UT2J, Toulouse, France \\ Email: jroux@laas.fr
}

How to cite this paper: Roux, J., Escriba, C., Fourniols, J.-Y. and Soto-Romero, G. (2019) A New Bi-Frequency Soil Smart Sensing Moisture and Salinity for Connected Sustainable Agriculture. Journal of Sensor Technology, 9, 35-43.

https://doi.org/10.4236/jst.2019.93004

Received: February 9, 2019

Accepted: September 27, 2019

Published: September 30, 2019

Copyright ( 2019 by author(s) and Scientific Research Publishing Inc. This work is licensed under the Creative Commons Attribution International License (CC BY 4.0).

http://creativecommons.org/licenses/by/4.0/

\begin{abstract}
Optimizing water consumption is a major challenge for more sustainable agriculture with respect for the environment. By combining micro and nanotechnologies with the offered solutions of IoT connection (Sigfox and LoRa), new sensors allow the farmer to be connected to his agricultural production by mastering in real time the right contribution needed in water and fertilizer. The sensor designed in this research allows a double measurement of soil moisture and salinity. In order to minimize the destructuring of the ground to insert the sensor, we have designed a cylindrical sensor, easy to insert, with its electronics inside its body to propose a low power electronic architecture capable of measuring and communicating wireless with a LoRa or Sigfox network or even the farmer's cell phone. This new smart sensor is then compared to the current leaders in agriculture to validate its performance. Finally, the sensor has better performance than commercials, a better response time, a better precision and it will be cheaper. For the salinity measure, it can detect the level of fertilizer in the soil according to the need of farmers.
\end{abstract}

\section{Keywords}

Smart Sensing, Connected Agriculture, Bi-Frequency Sensor

\section{Introduction}

In the context of agriculture modernization, the farmers need tools to develop the smart farming [1] [2]. For that, it is necessary to develop new sensors which can be deployed closest to the plants. To control the irrigation, the sensors measure the soil moisture near the crops [3] [4]. This new intelligent sensor combines non-contact moisture and salinity measurement [5], exploiting a ca- 
pacitive reading between two spiral form factor electrodes on a cylindrical preform to optimize contact surfaces for minimal electrode volume. Thus, by reducing the cost of the measuring point, it is possible to deploy more sensors in the ground and thus obtain an observation in the field more in agreement with the variations of the behavior of the grounds. The two optimized parameters are, on the one hand, soil moisture, which measured at different depths allows to know the mechanism of absorption of the crop plant; and on the other hand, the salinity of the soil which gives information on the amount of soil nutrients necessary for the development of the plant [6] [7].

In order to reduce the cost of manufacture and the constraints of placement in the ground, we did not retain the resistive measurement by contact [8] [9] [10] but privileged a capacitive measurement. The main advantage of this type of sensor is that it possesses a response time of less than a minute, which gives it the ability to monitor the hydric condition of the soil in close to real time [11]. Existing solutions [12] [13] [14] include sensors based on a small-sized $(<1 \mathrm{~mm})$ detection cell), which limits the volume of soil that can be tested. On the other hand, their complex structures [15] [16] [17] do not make them easy to use and require some time, several months, to restructure the soil, which can be damaging to farmers. Moreover, some commercials (Enviroscan, Meteor) is available but their costs limit their deployment and do not permit a reliable cover of the fields or others (Divine) can't be implemented for a season of culture and provide just a portable measurement.

The article presented will be oriented in three parts. The form factor of the electrodes will not be detailed in order to privilege on the one hand the electronic architecture of reading bi-frequency retained, then we will demonstrate by experimentation the usable frequency bands to observe respectively salinity and humidity [18]. Finally, we will present the results of the assembled sensor and will position its measurement sensitivity with respect to Decagon ${ }^{\text {tw }}$ and Sentek ${ }^{\text {tm }}$ sensors used by the farmers.

\section{Sensor's Model}

We chose the capacitive method to measure so the sensor is a capacity. But because of the integration, parasite capacities are created as shown in Figure 1.
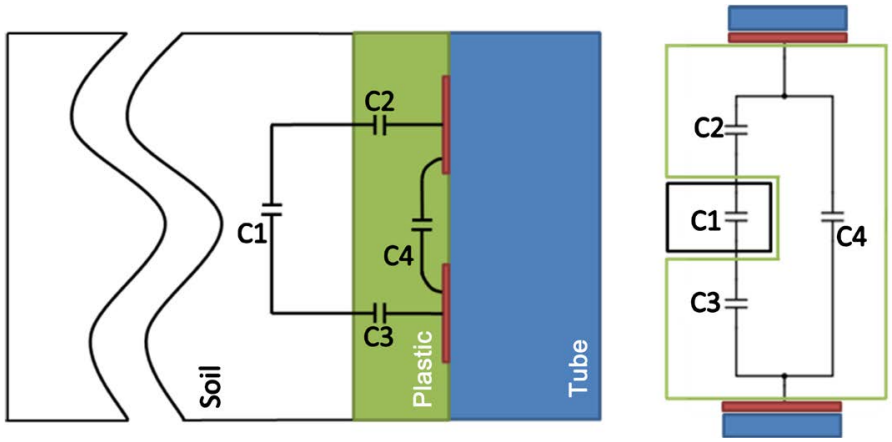

Figure 1. Electric model of the sensor. 
In this model, we can see the variable capacity $\mathrm{C} 1$ dependent to soil properties (humidity or salinity) and also fixed capacities created by the electrodes' interferences $\mathrm{C} 4$ and the plastic protection $\mathrm{C} 2$ and $\mathrm{C} 3$. This model can be simplified by a single variable capacity in parallel with a single fixed capacity.

\section{Measurement Architecture}

The capacitive variation induced by the lining of soil properties in response to a variation of humidity and/or salinity is exploited by a Colpitts oscillator which generates a sinusoidal signal of adjustable frequency to sweep the spectrum of measurements to search for the spectral band containing the information. In Figure 2, we can see the schematic of this Colpitts oscillator and its output frequency that is inversely proportional to the soil moisture.

The oscillation frequency can be read by an analog acquisition chain (Figure 3) associating an integrated frequency/voltage converter and a voltage reading using a CAN built into a microcontroller. Therefore, thinking microcontroller forces us to compare this architecture with an all-digital solution (Figure 4) with a dedicated embedded algorithm.

Three levels of salinity have been defined; the frequency sweep performed by the Colpitts oscillator shows the variation of capacity on the interval $100 \mathrm{kHz}$ to
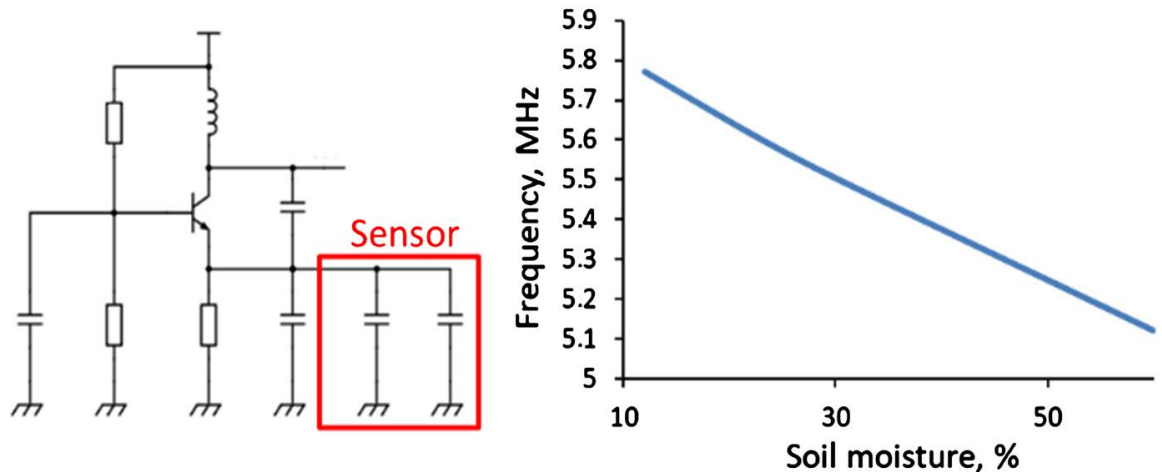

Figure 2. Frequency response of the Colpitts oscillator by the soil moisture.

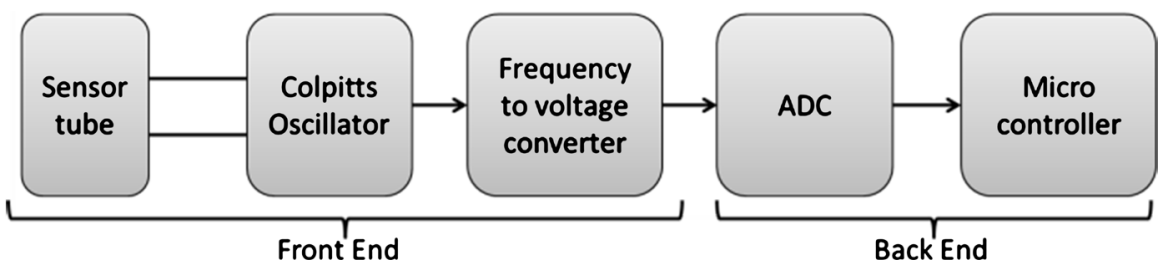

Figure 3. Analog sensor's architecture.

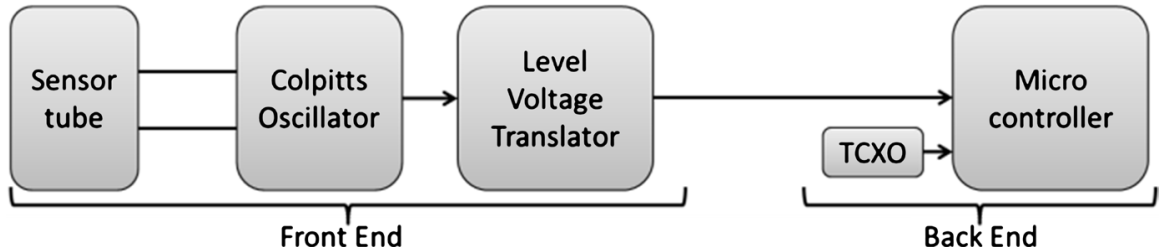

Figure 4. Digital sensor's architecture. 
$10 \mathrm{MHz}$ (Figure 5).

Two areas of interest appear on this curve:

- An area where the capacitance variation of the electrodes is insensitive to the salinity: above $4 \mathrm{MHz}$, the curves merge regardless of the salinity.

- An area where the capacity varies proportionally with salinity. Thus, the measurement of the salinity is defined at the frequency of $500 \mathrm{kHz}$.

By observing (Figure 6) the variation of capacity as a function of humidity, the reading frequency ranges are not superimposed with the exploitable area of the $500 \mathrm{kHz}$ dedicated to the salinity measurement. We can, therefore, define 8 $\mathrm{MHz}$ frequency for humidity measurement and thus obtain two operating ranges of our electrodes that will de-worm two totally uncorrelated salinity and soil moisture observations.

To make the good choice between an analogue or all-digital architecture, it is necessary to compare the stabilization of the measurement with the variations of environment of which one of the principal parameters is the variation of temperature in the soil which varies between $5^{\circ} \mathrm{C}$ to $50^{\circ} \mathrm{C}$ (Figure 7). While the time

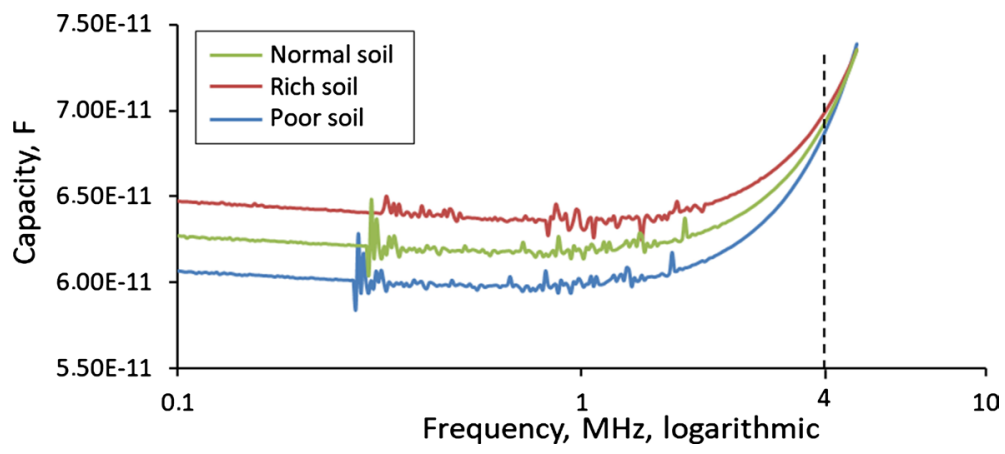

Figure 5. Sensor's capacity by the soil salinity.

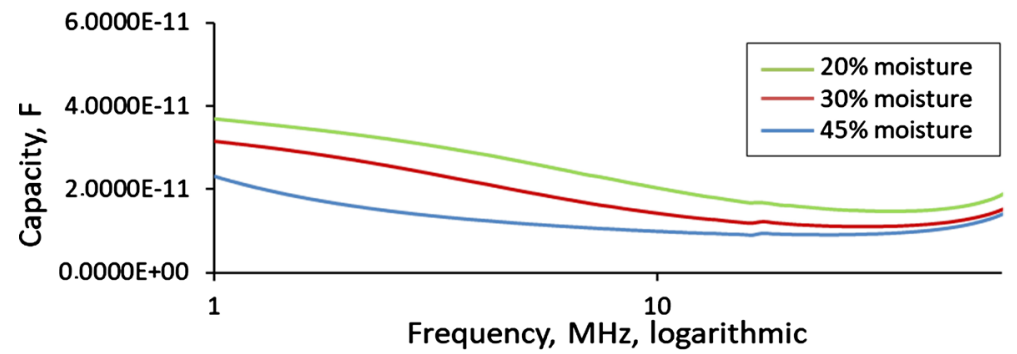

Figure 6. Sensor's capacity by soil moisture.

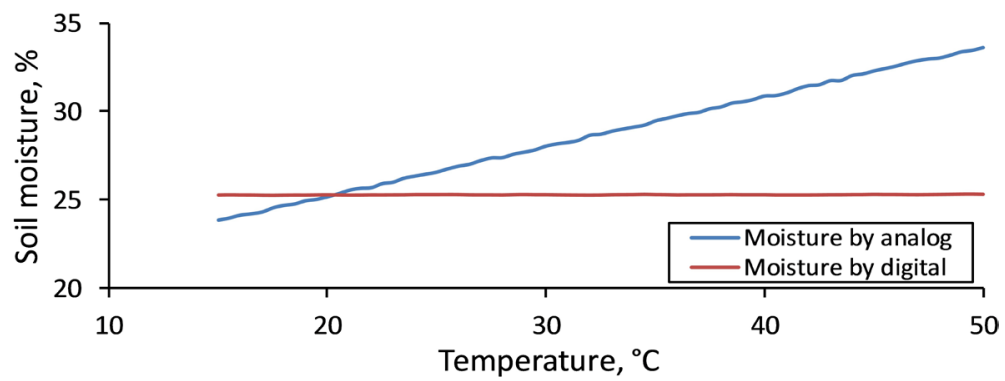

Figure 7. Thermal response of both architectures. 
frequency converter has at a sensitivity of $0.3 \% \cdot{ }^{\circ} \mathrm{C}^{-1}$ for the analog architecture; the TCXO choice allows the digital architecture to guarantee robustness to temperature variations. With this quartz cost constraint, all digital solution is chosen and can offer versatile corrections linked to soil characteristics variations (type of soil size of aggregates...).

\section{Sensor Integration}

To allow the insertion in the ground and to ensure the solidity, we decide to place the electronic card in the sensor tube even if the presence of components close to the electrodes will modify the electromagnetic behavior. The contact between the electrodes of the tube and the sensor is made using contact zones positioned on the top of the card. Connections with the outside (data + energy) are made on the top of the card and are solidified by cable holes. Complete integration is shown in Figure 8 with its characteristics in Table 1.

To be available by the farmers, the sensor's data have to be online. For that, the sensor has to be connected. Several links exist but two of them stand out, Sigfox and LoRa. These protocols are low rate but long range and low energy. With these links, the sensor can transmit at more than $10 \mathrm{~km}$ while remaining autonomous.

The sensor is buried on the soil so it cannot emit itself, he needs a part aboveground. This part concentrates the data of four sensors to measure at four depths. It will transmit the data to a server and a web application posts them to the farmers.

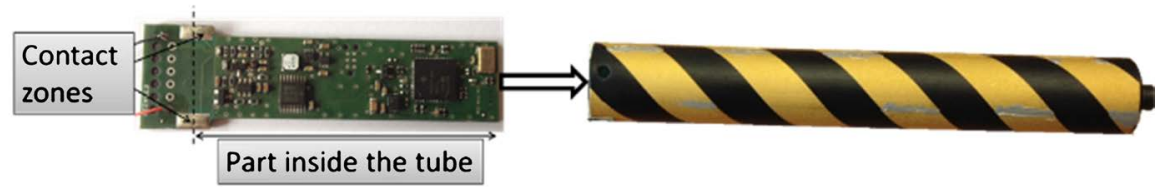

(a)

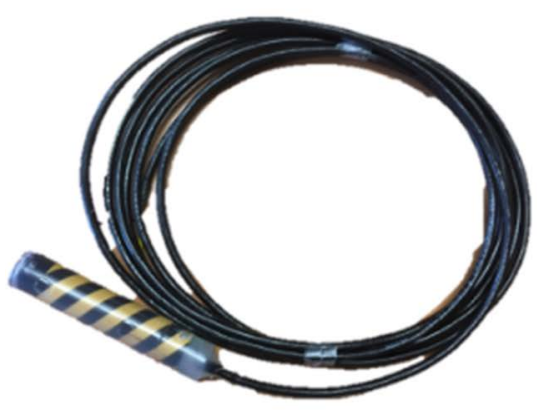

(b)

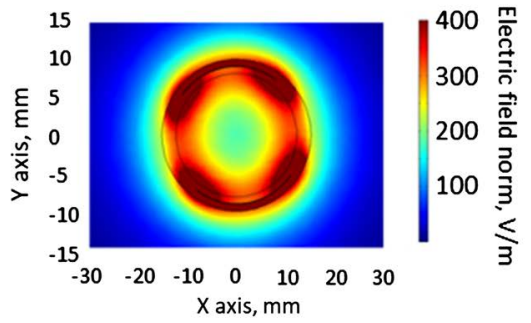

(c)

Figure 8. Sensor integration

Table 1. Our sensor's performance compared to market leader.

\begin{tabular}{ccccc}
\hline Sensor & $\begin{array}{c}\text { Precision } \\
(\% \text { moisture })\end{array}$ & $\begin{array}{c}\text { Measuring } \\
\text { time }(\mathrm{ms})\end{array}$ & Supply & Size \\
\hline Our sensor & $\pm 2 \%$ & 15 & $3 \mathrm{~V}-5 \mathrm{~V} @ 15 \mathrm{~mA}$ & $\emptyset 24 \times 150$ \\
DecagonTM & $\pm 2 \%$ & 20 & $2.5 \mathrm{~V}-3.6 \mathrm{~V} @ 10 \mathrm{~mA}$ & $20 \times 50$ \\
SentekTM & $\pm 2 \%$ & 50 & $12 \mathrm{~V} @ 100 \mathrm{~mA}$ & $\varnothing 40 \times 500$ \\
\hline
\end{tabular}




\section{Laboratory Tests}

To reproduce field conditions in laboratory, we sample soil in culture. This soil is heated to eliminate all water contained. To evacuate all the water, the soil is weighed before and after the heated phase. If the weight does not change, the soil is dry. If not, a new phase is begun. Then the soil is separated in calibrated samples.

To obtain a precise moisture range, a calibrated quantity of water relative to the weight of the sample is added to the samples to create different levels of moisture.

For the salinity, a calibrated quantity of fertilizer is added to the water but the sample receives the same quantity of water.

The samples are stocked on airtight jar to avoid evaporation and then modifications of the samples.

To realize the measurement, we buried the sensor in the jar. As the pot is made of glass, the electromagnetic field does not distribute and the measure is correct (Figure 9). We use an impedance-meter which can sweep the frequency of measurement to obtain the results presented in this paper.

Then, Figure 10 shows the sensor measuring salinity variations. To obtain

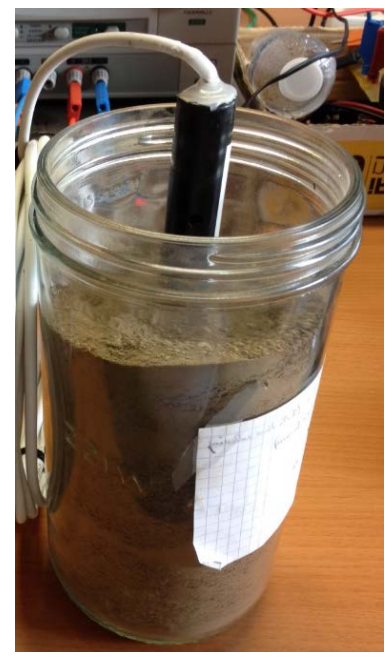

Figure 9. Measurement in a jar.

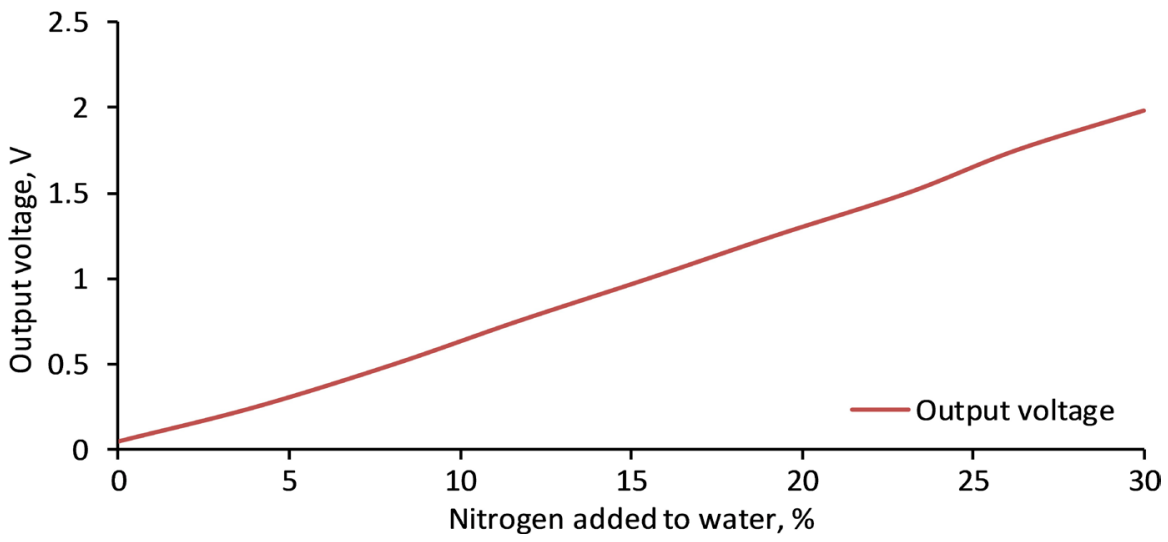

Figure 10. Sensor's output by soil salinity. 
these variations, soil samples are moistened to the same humidity but in addition, nitrogen, a common fertilizer, is dissolved to modify soil salinity.

We can observe that output can be approached by the following linear relation:

$$
V_{s}(V)=0.0657 * \text { Nitrogenadded }(\%)-0.0023
$$

In fact, the sensor has not to be very precise. The farmers set a threshold below which it is needed to add fertilizer. The threshold changes with the type of culture. The salinity measurement is validated.

\section{Sensor Measurements}

Observe and compare the behavior of our sensor over long periods with industrialized sensors on an orchard and cornfield culture (Figure 11 \& Figure 12). Look at the moisture response following the water supplies confirmed by the rain gauge. We can notice that we are more precise on the observation of the soil drying dynamics.

We can observe on this graph the similarity of response between the Decagon $^{\text {tw }}$ and our sensor: response time to a water intake is immediate; drying dynamics are identical. In addition, note in the purple box area: our sensor detects

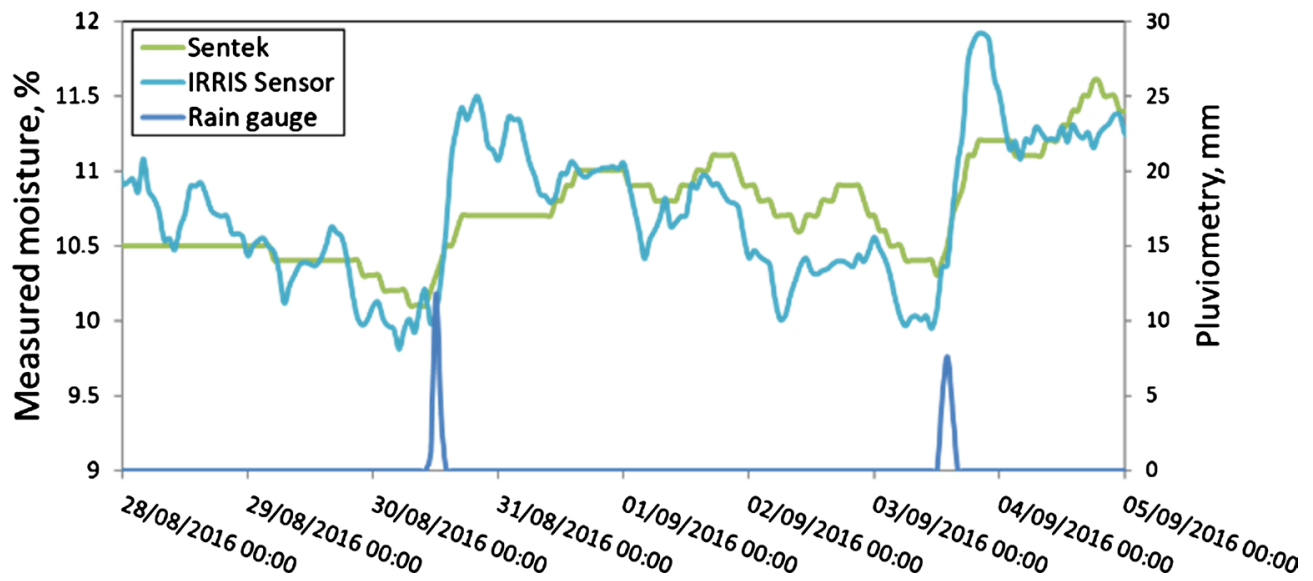

Figure 11. Cornfield culture sensing.

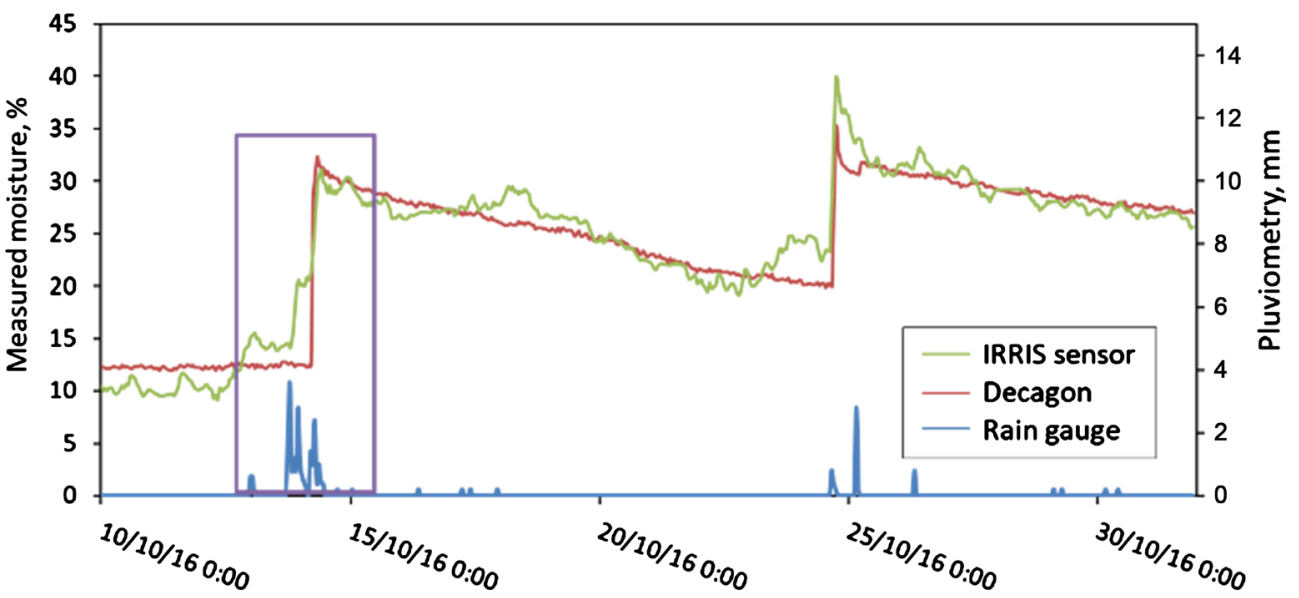

Figure 12. Apple orchard sensing. 
a water intake not seen by the Decagon ${ }^{\mathrm{rm}}$ sensor, which, in this example, reflects a better sensitivity.

\section{Conclusions}

Using capacitive technology for our sensor, we develop a new smart sensor able to measure soil moisture and also soil salinity. For this purpose, double helix electrodes are formed to optimize the relationship between the sensor and the ground. Bases on Colpitts oscillator we develop dual-frequency electronics and a full digital signal processing to reduce cost. Regarding the humidity measurement, we obtain a sensor that has the same performance as the market leaders but for lower cost and a new functionality.

Now for a large deployment, new tests have to be made to test the resistance of the sensor. For the tests, even if they were realized in actual exploitations, they were not manipulated by farmers. With these tests, a new mechanical shape may be designed. Moreover, these tests will permit to monitor the plastic protection's usury. Our longer test lasts 6 months so we don't know if the sensor can be reused several seasons of culture.

\section{Acknowledgements}

This work was realized within the framework of the IRRIS project which is funded by the French Midi Pyrenees council and the French Ministry of Agriculture. Great thanks are due to TCSD (Telecommunication Service \& Distribution) who lead the project and helped us to deploy our system into actual agricultural exploitation. We would thank Gilbert SA for their industrial support and their advice for the mechanical integration.

\section{Conflicts of Interest}

The authors declare no conflicts of interest regarding the publication of this paper.

\section{References}

[1] Nabayi, A., The, C.B.S., Mohd Hanif, A.H. and Sulaiman, Z. (2018) Plant Growth, Nutrient Content and Water Use of Rubber (Hevea brasiliensis) Seedlings Grown Using Root Trainers and Different Irrigation Systems. Pertanika Journal of Tropical Agricultural Science, 41, 251-270.

[2] Pigford, A.-A.E., Hickey, G.M. and Klerkx, L. (2018) Beyond Agricultural Innovation Systems? Exploring an Agricultural Innovation Ecosystems Approach for Niche Design and Development in Sustainability Transitions. Agricultural Systems, 164, 116-121. https://doi.org/10.1016/j.agsy.2018.04.007

[3] Gao, Z., Zhu, Y., Liu, C., Qian, H., Cao, W. and Ni, J. (2018) Design and Test of a Soil Profile Moisture Sensor Based on Sensitive Soil Layers. Sensors, 18, 1648. https://doi.org/10.3390/s18051648

[4] Faybishenko, B. (2000) Tensiometer for Shallow and Deep Measurements of Water Pressure in Vadose Zone and Groundwater. Soil Science, 165, 473-482. https://doi.org/10.1097/00010694-200006000-00003 
[5] Visacro, S., Alipio, R., Murta Vale, M.H. and Pereira, C. (2011) The Response of Grounding Electrodes to Lightning Currents: The Effect of Frequency-Dependent Soil Resistivity and Permittivity. IEEE Transactions on Electromagnetic Compatibility, 53, 401-406. https://doi.org/10.1109/TEMC.2011.2106790

[6] Oriol, P., Rapanoelina, M. and Gaudin, R. (1995) Le pilotage de l'irrigation de la canne à sucre par tensiomètres. Agricultural Development, 6, 39-48.

[7] Moutonnet, P., Brandy-Cherrier, M. and Chambon, J. (1981) Possibilites d'utilisation des tensiometres pour l'automation de l'irrigation. Plant Soil, 59, 335-345. https://doi.org/10.1007/BF02184205

[8] Zhou, Q.Y., Shimada, J. and Sato, A. (2001) Three-Dimensional Spatial and Temporal Monitoring of Soil Water Content Using Electrical Resistivity Tomography. Water Resources Research, 37, 273-285. https://doi.org/10.1029/2000WR900284

[9] Dean, T.J., Bell, J.P. and Baty, A.J.B. (1987) Soil Moisture Measurement by an Improved Capacitance Technique, Part I. Sensor Design and Performance. Journal of Hydrology, 93, 67-78. https://doi.org/10.1016/0022-1694(87)90194-6

[10] da Costa, E., de Oliveira, N., Morais, F., Carvalhaes-Dias, P., Duarte, L., Cabot, A. and Siqueira Dias, J. (2017) A Self-Powered and Autonomous Fringing Field Capacitive Sensor Integrated into a Micro Sprinkler Spinner to Measure Soil Water Content. Sensors, 17, 575. https://doi.org/10.3390/s17030575

[11] Chakraborty, M., Kalita, A. and Biswas, K. (2018) PMMA-Coated Capacitive Type Soil Moisture Sensor: Design, Fabrication, and Testing. IEEE Transactions on Instrumentation and Measurement, 68, 189-196. https://doi.org/10.1109/TIM.2018.2838758

[12] Kalita, H., Palaparthy, V.S., Baghini, M.S. and Aslam, M. (2016) Graphene Quantum Dot Soil Moisture Sensor. Sensors and Actuators B: Chemical, 233, 582-590. https://doi.org/10.1016/j.snb.2016.04.131

[13] Palaparthy, V.S., Kalita, H., Surya, S.G., Baghini, M.S. and Aslam, M. (2018) Graphene Oxide-Based Soil Moisture Microsensor for in Situ Agriculture Applications. Sensors and Actuators B: Chemical, 273, 1660-1669. https://doi.org/10.1016/j.snb.2018.07.077

[14] Boudaden, J., Steinmal, M., Endres, H.E., Drost, A., Eisele, I., Kutter, C. and MüllerBuschbaum, P. (2018) Polyimide-Based Capacitive Humidity Sensor. Sensors, 18, 1516. https://doi.org/10.3390/s18051516

[15] Xiao, D., Feng, J., Wang, N., Luo, X. and Hu, Y. (2013) Integrated Soil Moisture and Water Depth Sensor for Paddy Fields. Computers and Electronics in Agriculture, 98, 214-221. https://doi.org/10.1016/j.compag.2013.08.017

[16] Dias, P.C., Cadavid, D., Ortega, S., Ruiz, A., França, M.B., Morais, F.J.O., Ferreira, E.C. and Cabot, A. (2016) Autonomous Soil Moisture Sensor Based on Nanostructured Thermosensitive Resistors Powered by an Integrated Thermoelectric Generator. Sensors and Actuators A: Physical, 239, 1-7. https://doi.org/10.1016/j.sna.2016.01.022

[17] Pichorim, S., Gomes, N. and Batchelor, J. (2018) Two Solutions of Soil Moisture Sensing with RFID for Landslide Monitoring. Sensors, 18, 452. https://doi.org/10.3390/s18020452

[18] Weil, N.C., Brady, R.R. and Weil, R.R. (2016) The Nature and Properties of Soils. Fifteenth Edition, Pearson, Columbus. 